\title{
Prácticas y acciones de investigación y extensión presentes en el currículo de la Facultad de Agronomía, Universidad Nacional Agraria
}

\section{Practices and processes of research and extension found at the curriculum of agronomy school, Universidad Nacional Agraria}

Freddy Alemán-Zeledón', Sandra María Lovo-Jerez²

${ }^{1}$ Ingeniero Agrónomo, Docente Facultad de Agronomía, UNA, freddy@ci.una.edu.ni

${ }^{2}$ Ingeniero Agrícola, Dirección de Investigación, Extensión y Posgrado DIEP

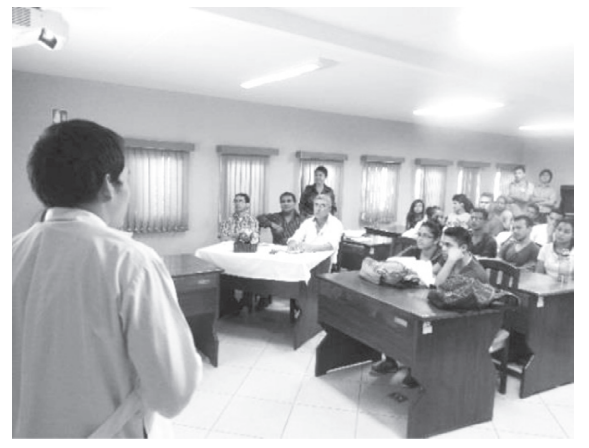

\section{RESUMEN}

La efectiva vinculación entre docencia, investigación y extensión es una necesidad en la formación del profesional de la Universidad Nacional Agraria. En el periodo de julio a noviembre del año 2014, se llevó a cabo un estudio con el propósito de obtener información acerca de prácticas y acciones curriculares y extracurriculares presentes en el currículo de la Facultad de Agronomía de la Universidad Nacional Agraria que fomentan la investigación y la extensión. La investigación es de tipo cuantitativa, no experimental. Para recolectar información se hizo uso de la técnica de revisión documental y encuestas, se aplicaron cuestionarios a docentes y estudiantes de los dos últimos años de las carreras de la Facultad de Agronomía. Los resultados muestran que los mecanismos más difundidos en la FAGRO-UNA para potenciar las capacidades en investigación y extensión son jornadas universitarias, planes emergentes, y prácticas pre-profesionales. Los estudiantes desconocen acerca de algunas de las prácticas que fomentan la investigación y la extensión, tales como movimientos de alumnos monitores y emprendedores, en cambio, se identifican con jornadas científicas y prácticas pre-profesionales. El currículo de la FAGRO-UNA debe incluir planes de investigación formativa, movimientos de emprendedores, pasantías y jornadas científicas, estudiantes de alto rendimiento, y fomentar desde los años iniciales de las carreras prácticas extracurriculares que permitan mayor alcance de las funciones en estudio. Las actividades curriculares de mayor impacto en la formación en investigación y extensión son giras de campo y establecimiento de cultivos, necesitando enfatizar en prácticas de laboratorios y la convivencia directa con la familia productora. El ajuste a los currículos de la FAGRO-UNA permitirá que la investigación y la extensión permeen el currículo, logrando con ello estudiantes creativos, analíticos, que los conviertan en agentes de cambio para la sociedad nicaragüense. Palabras clave: competencias, investigación, eje curricular, vinculación docencia-investigación-extensión, innovación educativa, aprendizaje para la vida.

\begin{abstract}
An effective link between teaching, research, and extension is a necessity in the professional training of Universidad Nacional Agraria. In the period from July to November 2014, a study was carried out with the purpose of gather information about curricular and extracurricular practices and actions of research and extension present at the curriculum of Agronomy Faculty, Universidad Nacional Agraria. The research was quantitative, not experimental. The information was collected using documentary review and surveys. Questionnaire were applied to teachers and students of the last two years at the Faculty of Agronomy. The results show that at FAGRO the most widespread mechanisms to enhance capabilities in research and extension are scientific university journeys, emerging plans, and pre-professional practices. Students do not know about some of the practices that encourage research and extension, such as teaching assistant and entrepreneurs, however, they are identified with scientific conferences and pre-professional practices. The FAGRO curriculum should include formative research plans, movements of entrepreneurs, internships and scientific conferences, high-achieving students, and encouraging from the early years of the careers extracurricular practices that allow greater scope of research and extension. Curricular activities of greater impact in research training and extension are field trips and crop establishment, and needs to emphasize in laboratory practice and direct coexistence with the farm family. The adjustment to the study plan of the agronomy faculty will allow research and extension permeate the curriculum, thereby achieving creative, analytical students, who become agents of change for Nicaraguan society.
\end{abstract}

Recibido: 7 de junio 2017

Aceptado: 25 de octubre del 2017

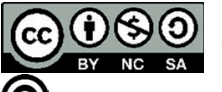

Los artículos de la revista La Calera de la Universidad Nacional Agraria, Nicaragua, se comparten bajo términos de la licencia Creative Commons: Reconocimiento, No Comercial, Compartir Igual. Las autorizaciones adicionales a las aquí delimitadas se pueden obtener en el correo freddy.aleman@ci.una.edu.ni Universidad Nacional Agraria 


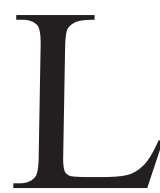

a investigación y la extensión son prácticas presentes en el quehacer de la Universidad Nacional Agraria (UNA). La misión de la UNA establece estas funciones como componentes fundamentales del accionar de la institución, a través de las cuales se genera conocimiento para la sociedad nicaragüense y se resuelven problemas en los ámbitos productivo y ambiental en pro del bienestar de la sociedad nicaragüense.

A pesar de cumplir con los aspectos mencionados, las funciones de investigación y extensión deberían ser componentes integrales del currículo de las carreras de grado de la Universidad Nacional Agraria. A la fecha, la discusión dentro de la Universidad ha girado en torno al papel que deben de cumplir estas funciones para retroalimentar el currículo y de esa forma contribuir a la formación integral de los estudiantes.

Al respecto, De Castilla (2008), manifiesta que la gestión universitaria tiene un reto mayúsculo, y es entender la investigación y la extensión como procesos integrados al currículo. La investigación universitaria, además de ofrecer los conocimientos y la información necesaria para el desarrollo nacional, participa sustantiva y decisivamente de la función educativa, que es la función fundamental de toda institución de educación superior. Este autor enfatiza que se deben crear las condiciones en las Universidades para el surgimiento y desarrollo del espíritu científico-investigativo en las comunidades universitarias.

Para Vargas (2010), el instituir la investigación y la extensión como ejes curriculares viene determinada por la necesidad que los participantes se conviertan en sujetos de cambio social. Para este autor, los docentes deben contribuir a este logro, a través de la revisión de sus prácticas y a la percepción de las competencias que se requieren para investigar y trasferir el conocimiento. Por otro lado, construyendo y transfiriendo conocimientos y permitiendo que los sujetos los construyan y transfieran, estarían formando los profesionales integrales que requiere el país.

En la UNA es a partir del año 2007, en el marco del programa Universidad emprendedora (2007-2010), iniciativa del Consejo Nacional de Universidades, se instruyó la discusión sobre muchos aspectos del quehacer universitario, entre ellos, la forma cómo los resultados de las investigaciones permean el currículo de grado que ofrece la institución, en pro de una formación integral de los educando (CNU, 2012). González (2006), esboza el papel que debe cumplir la investigación en el aprendizaje de los saberes, es decir, los procesos investigativos modelados didácticamente, para llevar a las clases las prácticas históricas de producción del conocimiento científico en sus procesos y no sólo en sus resultados, como un primer acercamiento del estudiante al mundo de la producción de conocimiento científico.

El currículo actual de la UNA carece de asignaturas que fomenten la investigación formativa y el emprendimiento, se orienta principalmente a la formación técnica del estudiante y a crear condiciones para la presentación y defensa del trabajo de diploma, sin considerar que esta actividad debería ser orientada a la formación de un individuo crítico, analítico y que resuelva problemas de forma creativa e innovadora.
En vista de lo antes expuesto se llevó a cabo el presente estudio con el propósito de generar información acerca de prácticas y acciones de investigación y extensión presentes en el plan de estudio de las carreras de la Facultad de Agronomía (FAGRO) de la UNA que a juicio de docentes y estudiantes retroalimentan el currículo y contribuyen a la formación integral de los estudiantes.

\section{METODOLOGÍA}

Tipo de investigación. La investigación es de tipo cuantitativa, no experimental. Incluye revisión documental y encuestas para docentes y estudiantes.

Localización del estudio. El estudio se realizó en el campus de la Universidad Nacional Agraria. Se visitaron en sus aulas de clases a estudiantes de los últimos años de las carreras de FAGRO, a quienes se les aplico un instrumento. De igual forma a través de correo electrónico se enviaron los formularios a docentes, obteniendo respuesta del 50 por ciento de ellos. Los restantes profesores fueron visitados directamente en sus oficinas para el llenado del formulario.

Registro de la información. El registro de la información se realizó a través revisión documental y de técnicas cuantitativas. A través de la revisión documental se pudo conocer aspectos de investigación y extensión que se encuentren recogidos en documentos normadores del quehacer académico de la Universidad Nacional Agraria, y como estos aspectos se encuentran permeando el currículo de la institución. Las encuestas, en el caso de los estudiantes, permitieron conocer acerca de las prácticas involucradas en la formación de los estudiantes que permiten el desarrollo de habilidades en investigación y extensión. En el caso de los docentes, se llevaron a cabo encuestas orientadas a conocer mecanismos implementados por los docentes para incluir investigación y extensión en el currículo, y aquellas prácticas que a juicio de los docentes deberían implementase para que la investigación y la extensión se conviertan en ejes transversales del plan de estudio.

Población y selección de la muestra. Para la selección de la muestra se aplicó la formula (selección de la muestra basada en el conocimiento que tenemos de la población) (Aguilar-Barojas, 2005). Para el caso de los docentes, la muestra fue de 28 académicos. Para el caso de los estudiantes la muestra fue de 72 estudiantes.

La selección de los docentes y los estudiantes se realizó a través de un muestreo aleatorio simple. Se obtuvo información sobre los estudiantes y docentes que conforman la población, se les número y a través de una tabla de números aleatorios se seleccionaran los sujetos de la muestra. Los estudiantes y docentes seleccionados fueron ubicados y se les administraron los instrumentos para la recopilación de datos. Se obtuvo información sobre los estudiantes pertenecientes a los IV y V años de las tres carreras pertenecientes a la facultad de agronomía (Agronomía, Protección Agrícola y Forestal e Ingeniería Agrícola para el Desarrollo Sostenible). 
Variables en estudio. Las variables evaluadas en el estudio fueron prácticas y acciones de investigación y extensión presentes en el currículo de las carreras. A cada una de las variables se le definieron indicadores para permitir su medición, y se propuso una técnica e instrumentos para colectar la información. Entre las variables destacan alumnos de alto rendimiento, investigación formativa, trabajos de curso, semilleros de investigación, prácticas de producción, prácticas de laboratorio, practicas pre-profesionales, movimientos de emprendedores, pasantías y alumnos monitores.

Análisis de la información. La información recopilada fue dispuesta en una base de datos. Inicialmente se realizó un análisis exploratorio de la información, para posteriormente analizarla utilizando cruce de la información. Se utilizó el software SPSS y se generaron graficas con los valores porcentuales de las respuestas obtenidas.

\section{RESULTADOS Y DISCUSIÓN}

Prácticas y acciones de investigación y extensión en el currículo de la Facultad de Agronomía. El análisis documental indica que en la FAGRO-UNA existen acciones de investigación y extensión como acciones extracurriculares. El análisis de los informes de rendición social de cuentas del CNU (CNU, 2012) muestra que anualmente se lleva a cabo la Jornada Universitaria de Desarrollo Científico, en la cual los estudiantes se involucran en investigaciones, la mayoría de ellas orientadas a graduación. Por otro lado, en los últimos cuatro años, como respuesta a mitigar los efectos de fenómenos naturales que afectan el sector rural nicaragüense, la UNA ha implementado los planes emergentes, por medio de los cuales estudiantes, después de ser capacitados trabajan directamente con las familias rurales llevando técnicas y tecnologías para mitigar los efectos negativos de dichos fenómenos.

La información revisada (modelo y proyecto educativo, plan estratégico institucional) no muestra iniciativas de investigación y extensión dentro del currículo. Aparte de las asignaturas orientadas a crear capacidades en los estudiantes para hacer investigación (metodología de la investigación, diseño de experimentos, seminario, modulo practico de experimentación agrícola), no existen mecanismos o programas institucionalizados de investigación formativa que beneficien a los estudiantes (UNA, 2011a, UNA, 2011b).

A pesar de algunas acciones extracurriculares, y en algunos casos eventuales, la FAGRO-UNA necesita incluir dentro del currículo de las carreras de grado elementos que permitan que la investigación y la extensión permeen el currículo. Estos elementos pueden ser estudiantes de alto rendimiento, planes de investigación formativa, movimientos de alumnos emprendedores, pasantías, jornadas científicas, etc.

En la FAGRO-UNA se hace necesaria una revisión exhaustiva de los contenidos de las asignaturas y retomar diversas acciones de investigación formativa y de visión empresarial que permita que los contenidos de las asignaturas desarrollen las herramientas que permitan la formación de estudiantes creativos, analíticos, que los conviertan en agentes de cambio para la sociedad nicaragüense.
Actividades curriculares y extracurriculares utilizadas por docentes de la UNA para incluir investigación y extensión. Los docentes de la facultad de agronomías implementan diversas actividades curriculares y extracurriculares con el propósito de incluir investigación y extensión en la práctica docente. El análisis de la información muestra que $78 \%$ de los docentes realizan actividades que fomentan la investigación y la extensión. La práctica más difundida es estudio de caso, seguido de giras de campo y trabajos de curso. En menor porcentaje se ubican prácticas de laboratorio y semilleros de estudiantes. Entre las prácticas no aparecen semilleros de investigación y estudiantes de alto rendimiento.

Las practicas descritas, más las actividades a nivel central (jornadas universitarias, planes emergentes, practicas pre-profesionales, etc.), se constituyen en los mecanismos de que dispone la Facultad de Agronomía para potenciar las capacidades de los estudiantes en investigación y extensión.

A pesar de la presencia de actividades de investigación y extensión en el plan de formación del estudiante, se hace necesario incluir actividades que permitan que la investigación y la extensión sean una práctica rutinaria en el plan de estudio, de uso común que sirva para la formación de individuos críticos, analíticos con herramientas suficientes para enfrentar un mercado de trabajo cada vez más competitivo. Al respecto, De Castilla (1998) indica que la investigación y la innovación deben constituirse en ejes transversales del currículum universitario alrededor del cual giren el accionar de las asignaturas y las prácticas docentes, tanto los métodos didácticos con los que se enseña, como los conocimientos que se procura enseñar.

Grado de satisfacción de los estudiantes con prácticas extracurriculares que incluyen investigación y extensión. La presencia de la investigación no se centra únicamente en que ésta esté presente en el currículo. Hay actividades extracurriculares que abonan a mejorar la formación del estudiante en investigación y extensión. Entre estos se mencionan los alumnos monitores, jornadas científicas, semilleros de investigación, los movimientos de emprendedores, entre otros (Osorio, 2008).

Se preguntó a los estudiantes acerca del grado de satisfacción con las prácticas extracurriculares que fomentan las capacidades de investigación y extensión en los estudiantes. El segmento mayor de estudiantes indica sentirse ni satisfecho, ni insatisfecho (50\%). En cambio, el 31 por ciento de los encuestados indica estar parcialmente y totalmente insatisfecho con dichas actividades. Un segmento menor, que incluye el 17 por ciento de los encuestados indica estar totalmente y parcialmente satisfecho

Algunas de las practicas evaluadas no tienen una difusión masiva dentro del estudiantado (movimiento alumnos monitores y movimiento de emprendedores), por tanto no toda la comunidad de estudiantes se siente identificado con ellas. Otras prácticas, como las jornadas científicas, alcanzan al segmento de estudiantes que se encuentra ejecutando su trabajo de investigación con fines de graduación. La única práctica generalizada entre los estudiantes de los últimos años son las prácticas pre-profesionales. 
Es necesario un mayor alcance de estudiantes con prácticas extracurriculares que fomente la investigación y la extensión. Debe fomentarse las jornadas científicas y ferias tecnológicas desde los años iniciales de las carreras, además, establecer las prácticas de producción y pre profesionales y con contenidos de investigación y extensión.

Practicas curriculares y extracurriculares que fomentan la investigación y extensión en los estudiantes de la FAGRO. En los currículos universitarios existen prácticas y acciones extra curriculares que permiten la investigación y la extensión permeen el currículo.

Al consultarle a los estudiantes acerca de las practicas extracurriculares y curriculares que ellos sienten fomentan el espíritu investigativo, encontramos que los grupos de investigación, prácticas de producción y alumnos monitores son consideradas de mayor impacto. Las ferias estudiantiles ocupan un lugar intermedio en la preferencia, en cambio, prácticas pre-profesionales, semilleros de investigación y estudiantes de alto rendimiento de acuerdo a la opinión de los estudiantes son las de menor impacto.

Los estudiantes de la FAGRO están familiarizados con las prácticas de producción y la investigación a través de grupos de investigación. Los mecanismos alumnos de alto rendimiento y semillero de investigación son prácticas de menor arraigo dentro de la UNA, lo cual limita el conocimiento de los estudiantes sobre estas prácticas de gran beneficio en otros sistemas de educación.

Los semilleros de investigación son una estrategia extra curricular de fomento a la investigación. Al respecto, Osorio, (2008) menciona que los semilleros de investigación buscan el desarrollo de competencias científicas e intelectuales capaces de articular docencia, investigación, y proyección social como tareas sustantivas para contribuir a la construcción y afianzamiento de los sujetos que aprenden en la sociedad del conocimiento.

En la UNA se necesita fomentar estrategias extracurriculares que provoquen y convoquen espacios para el desarrollo de nuevas competencias que promuevan el quehacer investigativo, que complementen y trasciendan los modelos tradicionales del aprendizaje.

Los docentes en su mayoría recalcan que las actividades de mayor impacto son giras de campo y establecimiento de cultivos, y que se necesita fortalecer las prácticas de laboratorios como también realizar la interacción con la familia productora.

Grado de satisfacción de los estudiantes con prácticas desarrolladas por los académicos para incluir investigación y extensión en la práctica docente. Por tradición, la formación Universitaria ha sido orientada a la formación técnica, obviando la promoción de una cultura emprendedora en los jóvenes que les permita una formación integral que conlleve la gestión de autoempleo y la generación de empleos para otros.

Al ser consultados los estudiantes acerca del grado de satisfacción que ellos sienten acerca de la práctica desarrollada por el docente para incluir investigación y extensión en la formación del estudiante, la mayoría expresa sentirse parcialmente satisfecho. Un segmento considerable manifiesta no estar ni satisfecho, ni insatisfecho (30 por ciento), $\mathrm{y}$ un 43 por ciento menciona estar totalmente y parcialmente satisfecho. El 27 por ciento de los encuestados manifiesta insatisfacción, 11 por ciento está totalmente insatisfecho, y 16 por ciento parcialmente insatisfecho.

La investigación y la innovación deben constituirse en ejes transversales del currículum universitario alrededor del cual giren el accionar de las asignaturas y las prácticas docentes, tanto los métodos didácticos con los que se enseña, como los conocimientos que se procura enseñar (De Castilla, 1998).

En la práctica, en la UNA existe una tendencia a la formación profesionalista de los estudiantes, lo que ocasiona que al egresar se ofrecen a un mercado de trabajo, careciendo de la formación suficiente que les permita ser ejecutores de su propia trasformación. Los egresados carecen de las competencias necesarias para ser gestores de sus propios ambientes de trabajo, y de esa forma tener las posibilidades de ser empleadores y no empleados.

Acceso a servicios y ambientes de aprendizaje en la FAGRO-UNA. Una universidad responsable debe proveer de facilidades a sus estudiantes para potenciar la práctica enseñanza-aprendizaje. Estos son laboratorios, ambientes propicios para el aprendizaje, cultura, deporte, áreas experimentales, etc. Al ser consultados los estudiantes acerca de la accesibilidad a servicios y ambientes de aprendizaje, el 60 por ciento de los encuestados manifestó estar satisfecho con dichos medios, en cambio 30 por ciento manifestó estar insatisfecho. Diez por ciento de la muestra manifestó desconocer sobre los aspectos consultados.

Los indicadores del Consejo Nicaragüense de Evaluación y Acreditación de la educación superior (CNEA) enfatizan sobre la necesidad de ambientes propicios para la práctica educativa. Considerando la opinión de los estudiantes, la FAGRO-UNA cuenta con las ambientes propicios para facilitar el aprendizaje de los estudiantes.

Actividades formativas por las cuales optan los estudiantes de la FAGRO-UNA. El currículo actual carece de asignaturas que fomenten la investigación formativa y el emprendimiento

Al ser consultados los estudiantes sobre las actividades formativas en las cuales mayoritariamente se involucran, se encontró que actividades deportivas trasciende como la actividad principal, seguido de investigación y actividades medioambientales. De acuerdo a la opinión de los estudiantes, las actividades de emprendimiento y extensión son con las cuales se sienten menos identificados.

La FAGRO-UNA necesita ir más allá de la formación técnica del estudiante, implementar actividades que fomente la investigación formativa del estudiante de tal forma que el producto sea individuos críticos, analíticos y que resuelva problemas de forma creativa e innovadora. 


\section{CONCLUSIONES}

El currículo de las carreras de grado de FAGRO-UNA debe incluir elementos que permitan que la investigación y la extensión permeen el currículo. Entre estos elementos están, estudiantes de alto rendimiento, planes de investigación formativa, movimientos de alumnos emprendedores, pasantías, jornadas científicas, entre otros.

Se hace necesario una revisión exhaustiva de los contenidos de las asignaturas y retomar diversas acciones de investigación formativa y de visión empresarial que permita la formación de estudiantes creativos, analíticos, que los conviertan en agentes de cambio para la sociedad nicaragüense.

Los mecanismos de que dispone la Facultad de Agronomía para potenciar las capacidades de los estudiantes en investigación y extensión son principalmente jornadas universitarias, planes emergentes y prácticas profesionalizantes,

La comunidad estudiantil desconoce acerca de algunas de las prácticas que fomentan la investigación y la extensión, entre ellas movimientos de alumnos monitores y movimiento de emprendedores, en cambio, se identifican con jornadas científicas y prácticas pre-profesionales.
Las prácticas extracurriculares que fomentan la investigación y la extensión deben poseer mayor alcance en los estudiantes. Las jornadas científicas y ferias tecnológicas deben fomentarse desde los años iniciales de las carreras, además, se deben establecer prácticas de producción y pre profesionales con contenidos de investigación y extensión.

Las actividades curriculares de mayor impacto en la formación en investigación y extensión son giras de campo y establecimiento de cultivo (módulos prácticos). Se hace necesario fortalecer las prácticas de laboratorios, y orientar la convivencia directa con la familia productora.

Los ambientes académicos para el desarrollo de investigación y extensión son propicios para facilitar el aprendizaje de los estudiantes.

Se deben crear las condiciones necesarias para fomentar la investigación formativa del estudiante de tal forma que el profesional agropecuario sea un individuo crítico, analítico y que resuelva problemas de forma creativa e innovadora.

\section{REFERENCIAS BIBLIOGRÁFICAS}

Aguilar-Baraojas, S. 2005.Formulas para el cálculo de la muestra en investigaciones de salud. Salud en Tabasco, 11(1-2), 333-338 CNU (Consejo Nacional de Universidades, NI). 2012. Informe rendición social de cuentas año 2012. Managua, Nicaragua: Consejo Nacional de Universidades.

.2012. Universidad Emprendedora en Nicaragua: Cultura de intercambio y aprendizaje para la Innovación (Primera ed.). (F. Alemán, \& S. Sheinberg, Edits.) Managua, Nicaragua: CNU.

De Castilla, M. 2008. La reforma Universitaria, Troya y mi idea de Universidad. Managua, Nicaragua: Lea Grupo Editorial. . 1998. La Universidad contra la Universidad. Managua: Universidad Nacional Autónoma de Nicaragua.

González, E. 2006. La investigación formativa como una posibilidad para articular las funciones universitarias de la investigación, la extensión y la docencia. Revista educación y pedagogía, XVIII (46), 101-109.

Osorio, M. 2008. La investigación formativa o la posibilidad de crear cultura investigativa en la educación superior: El caso de la práctica pedagógica de la licenciatura en educación básica con énfasis en humanidades, lengua castellana de la Universidad de Antioquia. Medellín: Universidad de Antioquia.

Vargas, S. 2010. Las competencias investigativas como eje curricular. Cuadernos de educación y desarrollo, 2(18), 1-4.

UNA (Universidad Nacional Agraaria, NI). 2011a. Modelo educativo (ME/UNA) y proyecto educativo. Managua, Nicaragua: Universidad Nacional Agraria

.2011b. Plan estratégico institucional (PEI, 2012-2016. Managua, Nicaragua: Universidad Nacional Agraria 